\title{
Bone modeling, remodeling, and skeletal health in children and adolescents: mineral accrual, assessment and treatment
}

\author{
Chiara Maggioli, MD, \\ Stefano Stagi, MD
}

Department of Health Sciences, University of Florence, Anna Meyer Children's University Hospital, Florence, Italy
Received: 22 March, 2017

Revised: 31 March, 2017

Accepted: 31 March, 2017

Address for correspondence:

Stefano Stagi, MD

Department of Health Sciences, University of Florence, Anna Meyer Children's University Hospital, viale Pieraccini 24, Florence, Italy

Tel: +39-055-5662405

Fax: +39-055-5662400

E-mail: stefano.stagi@yahoo.it

http://orcid.org/0000-0002-22594685
The modeling and remodeling process of the bone is fundamental to maintaining its integrity and mechanical properties. Many physical and biochemical factors during childhood and adolescence are crucially important for the development of healthy bones. Systemic conditions, such as hormonal status, nutrition, physical inactivity, or many pharmacological treatments, as well as a local variation in the load, can influence bone turnover and, consequently, the attainment of a proper peak bone mass. However, many diseases affecting children and adolescents can be associated with a reduction in bone accrual or a loss of bone mass and quality, which leads to an increased risk of fracture over one's life. In this review, we examine the effects of genetics, physical activity, chronic diseases and pharmacological treatments, and dietary factors on bone integrity in children and adolescents. We also briefly describe the specific tools that are currently used in assessing bone health.

Keywords: Child, Bone health, Adolescent, Bone density

\section{Introduction}

Osteoporosis in adults is one of the major causes of morbidity and mortality because of its association with fragility fractures ${ }^{1)}$. The peak bone mass (PBM) is well known as a key determinant of the lifetime risk of osteoporosis. In fact, childhood and adolescence are a critical time for bone development, mineralization, and the attainment of a $\mathrm{PBM}^{2}$. Therefore, osteoporosis prevention begins by optimizing bone mineral gains over an individual's growing years ${ }^{3)}$.

During childhood and adolescence, bone linear growth and bone mineral accrual proceed in different ways and velocities in various skeletal sites ${ }^{2}$. In particular, the growth of the appendicular skeleton is prevalent before puberty, whereas that of the spine mostly occurs later. During peripuberty, the areal bone mineral content (BMC) and bone mineral density (BMD) in the lumbar spine and proximal femur increase fourfold to sixfold. At the same time, the diaphyses of the long bone increase twofold ${ }^{4)}$.

Puberty is also the time of major gender differences in bone growth, particularly in terms of bone size and bone mass content. The bone mass accrual rate usually follows sex-specific patterns that are similar to those of height velocity but are delayed by about 6-12 months (Fig. 1). Therefore, the peripubertal period is characterized by relatively undermineralized bones and a higher risk of fractures until the PBM is achieved. In fact, the increase in bone mass and density carries on for several years after the end of linear growth. The exact time when bone accrual ceases is unknown, but it is estimated to be during the young adult age. In particular, it is specific for different sites: it ranges from 16 to 18 years for the spine and femoral neck, and up to 35 years for the skull ${ }^{4)}$. 

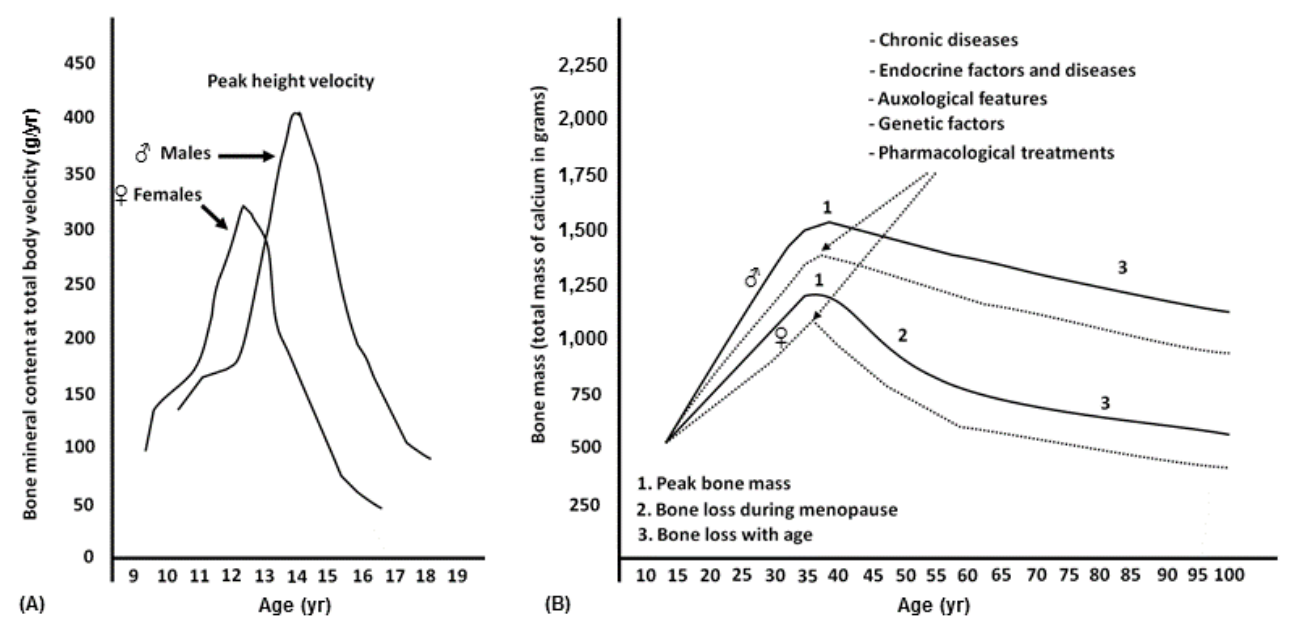

Fig. 1. (A) Growth of bone mass in relation to height growth velocity and difference in peak bone mass between males $\left({ }^{\circledR}\right)$ and females (ㅇ). (B) Difference in peak bone mass between males ( $\left.\delta^{7}\right)$ and females (ㅇ) and factors and diseases that influence the peak bone mass and the risk of osteopenia/osteoporosis in adulthood.

Among many factors that drive skeletal growth, the genetic factor plays a key role in the variability of $\mathrm{PBM}^{5}$. Genome-wide association studies have discovered numerous genes involved in this process. Polymorphisms in the genes encoding the vitamin D receptor, estrogen receptor, insulin-like growth factor-I, type I collagen, transforming growth factor beta, and interleukin 6 are the most intensively studied, but recently, some genes that also seem to influence BMD in children and osteoporosis in adults have been recognized. Aside from heritable factors, environmental factors, such as diet, physical activity ${ }^{6}$, and hormonal status, modify the complex mechanism of cellular interactions leading to bone accrual.

Multiple hormonal signals regulate bone growth and turnover. In addition to the well-known parathyroid hormone (PTH), calcitonin, thyroid hormones, the growth hormone, and sexual steroids, other hormones, cytokines, and growth factors with endocrine, paracrine, and autocrine actions have been the focus in an increasing number of studies. Bone cells and the skeletal matrix itself produce and contain many signals that influence bone growth; furthermore, neighboring muscle and adipose tissue exert significant 2-way interactions, with the skeleton contributing significantly to the final adult bone density ${ }^{7)}$. Particularly, as shown by recent studies, a higher muscle mass and greater brown adipose tissue volume are associated with a greater cortical bone density and thickness ${ }^{8}$.

\section{Definition}

Osteoporosis in childhood and adolescence is a condition characterized by a low BMD or BMC $(z$-score $\leq-2.0$, adjusted for age and gender) and the presence of a clinically significant fracture history".

\section{Causes}

Pediatric osteoporosis recognizes 2 pathogenetic pathways that can contribute to the development of this conditiondefects in bone accrual and mineralization, and excessive bone resorption. The causes of osteoporosis are included into 2 large groups, which identify primary and secondary osteoporosis. Primary osteoporosis is a rare disease that is mainly related to genetic defects leading to an intrinsic bone abnormality ${ }^{10)}$. Osteogenesis imperfecta, through its various clinical phenotypes, is the most common genetic disease. Fractures, blue sclera, a short stature, joint laxity, and hearing loss are typical features of osteogenesis imperfecta. Other genetic defects include some rickets, calcium and phosphorus metabolism alterations, and genetic syndromes, such as Marfan syndrome, Paget disease, and Ehler-Danlos syndrome (Table 1). Secondary osteoporosis in childhood is more frequent than primary osteoporosis (Table 2). Systemic chronic inflammatory diseases, malabsorption disorders, endocrinopathies, neoplastic diseases, and medications are the most common causes of secondary osteoporosis. Furthermore, prolonged immobilization can lead to a decrease in BMD, determining the reduction of mechanical stress on the bone. Among medications, many drugs can interfere with calcium and vitamin D absorption and metabolism, or adversely work directly on bone metabolism. Glucocorticoid-associated osteoporosis (GAO) is an iatrogenic condition that leads to an impaired BMD. Glucocorticoids work both on calcium metabolism and directly on bone cells. Particularly, calcium gastrointestinal absorption is impaired, and urinary excretion is increased. The effect on bone cells is characterized by a transient early phase of increased bone resorption through stimulation of osteoclasts. Conversely, in the long term, the major determinant of damage is impaired bone formation. Osteoblastogenesis is reduced, and apoptosis of osteoblasts and osteocytes is increased ${ }^{11}$. The osteoblastic 
Table 1. Causes of primacy osteoporosis in children and adolescents

\begin{tabular}{|c|c|}
\hline Disease & Gene \\
\hline Osteogenesis imperfecta & $\begin{array}{l}\text { COL1A1; COL1A2; IFITM5; SERPINF1; CRTAP; LEPRE1; PPIB; FKBP10; BMP1; SP7; SERPINH1; WNT1; } \\
\text { TMEM38B; SPARC }\end{array}$ \\
\hline X-linked hypophoshatemic rickets & PHEX; CLCN5 \\
\hline Homocystinuria & CBS; MTHFR \\
\hline Hypophosphatasia & ALPL \\
\hline Wilson disease & ATP7B \\
\hline Menkes' kinky hair syndrome & ATP7A \\
\hline Osteoporosis-pseudoglioma syndrome & LRP5 \\
\hline Idiopathic juvenile osteoporosis & - \\
\hline Juvenile Paget disease & $O P G$ \\
\hline Early-onset Paget disease & RANK \\
\hline Ehlers-Danlos syndrome & COL5A2; COL5A1; COL1A1; COL3A1;PLOD1; COL1A2; ADAMTS2; COL3A1; TNXB \\
\hline Bruck syndrome & FKBP10; PLOD2 \\
\hline Marfan syndrome & FBN1 \\
\hline Hypophosphatemic nephrolithiasis/osteoporosis & SLC34A1; NPHLOP2 \\
\hline Hajdu-Cheney syndrome & NOTCH2 \\
\hline Torg-Winchester syndrome & MMP2 \\
\hline Shwachman-Diamond syndrome & SBDS \\
\hline Singleton-Merten syndrome & IFIH1;DDX58 \\
\hline Schwartz-Jampel Syndrome & HSPG2 \\
\hline Cerebrooculofacioskeletal syndrome 1 & ERCC6 \\
\hline Cleidocranial dysostosis & RUNX2 \\
\hline Stuve-Wiedemann syndrome & LIFR \\
\hline Cole-Carpenter syndrome & P4HB; SEC24D \\
\hline Geroderma osteodysplasticum & GORAB \\
\hline Noonan syndrome & PTPN11;SHOC2; KRAS; SOS1; RAF1; NRAS; BRAF; RIT1 \\
\hline Neonatal hyperparathyroidism & CASR \\
\hline Other forms of hypophosphatemic rickets & SLC34A3; FGF23; DMP1; ENPP1 \\
\hline Hypocalcemic rickets & VDR;CYP2R1;CYP27B1 \\
\hline Turner syndrome & - \\
\hline Klinefelter syndrome & - \\
\hline Down syndrome & - \\
\hline Williams-Beuren syndrome & - \\
\hline
\end{tabular}

Table 2. Main conditions potentially causing an altered bone density and/or quality in childhood

\begin{tabular}{|c|c|}
\hline Classification & Disease \\
\hline Endocrine diseases & $\begin{array}{l}\text { Hypogonadism; insensitivity syndrome of estrogen; panhypopituitarism; growth hormone deficiency; hyperthyroidism; } \\
\text { Cushing syndrome; primary hyperparathyroidism; primary hypoparathyroidism; McCune-Albright syndrome }\end{array}$ \\
\hline latrogenic causes & $\begin{array}{l}\text { Anticonvulsants; gonadotropin-releasing hormone analogue; L-thyroxine (high dose); antiretroviral drugs; anticoagulants; } \\
\text { chemotherapeutic drugs; corticosteroids treatments }\end{array}$ \\
\hline Genetic syndromes & Turner syndrome; Klinefelter syndrome; 22q11 deletion syndrome; Down syndrome; Williams-Beuren syndrome \\
\hline Malignancies & Leukemia; lymphoma; solid tumors \\
\hline Nutritional problems & $\begin{array}{l}\text { Nervous anorexia; lactose intolerance; deficiency of calcium, copper, etc.; vegetarian diets; malnutrition; total parenteral } \\
\text { nutrition }\end{array}$ \\
\hline Chronic diseases & $\begin{array}{l}\text { Juvenile idiopathic arthritis; systemic lupus erythematosus; dermatomyositis; chronic renal failure; renal tubular acidosis; } \\
\text { idiopathic hypercalciuria; cholestatic forms; celiac disease; Crohn disease; ulcerative Colitis; congestive heart failure; } \\
\text { thalassemia; hereditary hemochromatosis; haemophilia; sickle cell anemia; systemic mastocytosis; hyper-lgE syndrome; } \\
\text { overweight/obesity }\end{array}$ \\
\hline Other & Immobilization/little use; intense physical activity; posttransplant; prematurity \\
\hline
\end{tabular}

function to stimulate the synthesis of type 1 collagen is also impaired. Moreover, glucocorticoid can alter bone metabolism through extra skeletal actions. Altered growth hormone synthesis and hypothalamic-pituitary-gonadal function are 
frequently involved in GAO. Because of accelerated muscle catabolism, glucocorticoid myopathy can have a role in bone damage. Furthermore, glucocorticoids can worsen bone damage, induced by an underlying inflammatory disease, and result in an increased risk of fractures. Prolonged inflammation indeed alters bone metabolism, which favors bone resorption in different ways. Interleukin 1 and interleukin 6 stimulate osteoclastogenesis through receptor activator of nuclear factor kappa-B ligand; therefore, collagen synthesis and osteoblast differentiation are impaired, and osteoblast apoptosis is increased $^{12,13)}$.

Malabsorption and celiac disease are the most common causes of bone damage. A low BMD and impaired bone growth in childhood are caused by reduced calcium and vitamin D absorption, as well as secondary hyperparathyroidism. In these patients, a strictly gluten-free diet significantly improves bone mineralization ${ }^{14)}$.

Many endocrinopathies affect bone health. Alterations in PTH metabolism, as well as thyroid hormones, the growth hormone, cortisol, and sex steroids, influence bone growth and accrual. Furthermore, type 1 diabetes impairs the attainment of the PBM, which increases the long-life risk of fractures ${ }^{15,16)}$.

The main mechanism underlying this association is a defect in osteoblast differentiation and activity because of many contributing factors, such as insulinopenia and pro-inflammatory cytokine production. Moreover, other mechanisms involved include hyperglycemia, oxidative stress, and the accumulation of advanced glycation end products that compromise collagen properties ${ }^{17)}$.

\section{Diagnosis}

A diagnosis of bone disease requires clinical assessment, laboratory, and imaging. Some conditions, such as primary osteoporosis, also need genetic investigation and bone biopsy. In this section, I describe the specific tools that are currently used in the diagnosis of osteoporosis.

Dual-energy X-ray absorptiometry (DXA) is a widely available technique that is used to investigate $\mathrm{BMC}$ and $\mathrm{BMD}^{9,18)}$. In association with a history of fractures, BMD evaluation is required to make a diagnosis of osteoporosis in children and adolescents. However, in pediatric patients, the correct reading of DXA is affected by several limitations. The T-score parameter is not applicable to children because it refers to the BMD value upon PBM achievement. The $z$-score is the index used in childhood and adolescence up to 20 years. It is adjusted for age, gender, and ethnic group, but it does not consider weight and height variability ${ }^{19}$. Therefore, in children with a short stature or delayed puberty, their BMD is underestimated. In fact, these patients have smaller bones compared with their peers. Unlike the T-score, the $z$-score without a fracture history cannot be used to characterize "osteoporosis," whereas the term "reduced bone density according to chronological age" should be used if the $z$-score is below -2.0 standard deviation, according to the International Society for Clinical Densitometry ${ }^{20}$.
Quantitative computed tomography (QCT) is an excellent alternative to DXA because it can evaluate cortical and trabecular compartments, as well as volumetric bone density. Highresolution QCT also assesses the microarchitecture of the bone and is useful in evaluating bone strength. However, the use of QCT is limited by its high cost and higher radiation than that of DXA $^{211}$.

Quantitative bone ultrasonometry (QUS) is a radiation-free approach to investigate bone mineral status in childhood. This method is fast to execute and cheap. Through measurements of the speed of sound, broadband ultrasound attenuation, stiffness factor, and bone transmission time, QUS can give information on not only density but also elasticity, microarchitecture, and thickness ${ }^{22,23)}$. However, at present, no evidence on the diagnostic accuracy of QUS in the pediatric population is available, although several studies have demonstrated its correlation with densitometric parameters. Therefore, in consideration of its characteristics, QUS cannot replace DXA, but it can be integrated to it.

\section{Therapeutical approaches}

The first approach to pediatric osteoporosis is prevention, if possible. This approach consists of identifying those patients at risk of bone disease and counseling them about lifestyle and dietary calcium and vitamin D supplementations. Several studies suggest that physical activity in childhood is an effective strategy to achieve the optimal $\mathrm{PBM}^{24}$. Secondary osteoporosis is treated by removing the underlying specific cause. The drugs approved for pediatric use in osteoporosis are limited. When the main mechanism of osteoporosis is bone resorption, treatment with an antiresorptive agent is the appropriate choice. Several studies demonstrated bisphosphonate utility in severe osteoporosis and in primary prevention in some conditions as glucocorticoid-induced osteoporosis, although this practice is not yet recommended ${ }^{25}$. Therefore, bisphosphonates are used in some genetic forms, such as osteogenesis imperfecta. The recombinant human parathyroid hormone, an anabolic agent that is effective in adults, is unavailable for children because of the risk of osteosarcoma. Indeed, this association with osteosarcoma is well demonstrated in rats in many studies, but it remains theoretical for humans ${ }^{26)}$.

\section{Conclusions}

Osteoporosis in childhood and adolescence is characterized by reduced bone density $(z$-score $\leq 2)$ associated with fragility fractures. This condition during an individual's growth years can alter bone mineral accrual, which leads to insufficient bone peak mass. It is a well-recognized risk factor for adult bone fragility. Primary osteoporosis is caused by genetic defects that lead to bone abnormality, whereas secondary osteoporosis results from multisystemic, often chronic, diseases, or their treatments. Prevention consists of a healthy lifestyle, physical 
activity, and optimal calcium and vitamin D intake. Therapy depends on the pathogenesis involved; in severe osteoporosis, bisphosphonates are recommended.

\section{Conflict of interest}

No potential conflict of interest relevant to this article was reported.

\section{References}

1. Javaid MK, Cooper C. Prenatal and childhood influences on osteoporosis. Best Pract Res Clin Endocrinol Metab 2002;16:349-67.

2. Bachrach LK. Acquisition of optimal bone mass in childhood and adolescence. Trends Endocrinol Metab 2001;12:22-8.

3. Carey DE, Golden NH. Bone health in adolescence. Adolesc Med State Art Rev 2015;26:291-325.

4. Stagi S, Cavalli L, Iurato C, Seminara S, Brandi ML, de Martino M. Bone metabolism in children and adolescents: main characteristics of the determinants of peak bone mass. Clin Cases Miner Bone Metab 2013;10:172-9.

5. Mitchell JA, Cousminer DL, Zemel BS, Grant SF, Chesi A. Genetics of pediatric bone strength. Bonekey Rep 2016;5:823.

6. Kambas A, Leontsini D, Avloniti A, Chatzinikolaou A, Stampoulis T, Makris K, et al. Physical activity may be a potent regulator of bone turnover biomarkers in healthy girls during preadolescence. J Bone Miner Metab 2016 Nov 12 [Epub]. https://doi.org/10.1007/s00774-016-0794-3.

7. Gordon CM, Zemel BS, Wren TA, Leonard MB, Bachrach LK, Rauch F, et al. The determinants of peak bone mass. J Pediatr 2017;180:261-9.

8. Ponrartana S, Aggabao PC, Hu HH, Aldrovandi GM, Wren TA, Gilsanz V. Brown adipose tissue and its relationship to bone structure in pediatric patients. J Clin Endocrinol Metab 2012;97:2693-8.

9. Bianchi ML, Baim S, Bishop NJ, Gordon CM, Hans DB, Langman CB, et al. Official positions of the International Society for Clinical Densitometry (ISCD) on DXA evaluation in children and adolescents. Pediatr Nephrol 2010;25:37-47.

10. Stagi S, Cavalli L, Seminara S, de Martino M, Brandi ML. The ever-expanding conundrum of primary osteoporosis: aetiopathogenesis, diagnosis, and treatment. Ital J Pediatr 2014;40:55.

11. von Scheven E, Corbin KJ, Stagi S, Cimaz R. Glucocorticoidassociated osteoporosis in chronic inflammatory diseases: epidemiology, mechanisms, diagnosis, and treatment. Curr Osteoporos Rep 2014;12:289-99.

12. Vega D, Maalouf NM, Sakhaee K. Clinical review \#: the role of receptor activator of nuclear factor-kappaB (RANK)/
RANK ligand/osteoprotegerin: clinical implications. J Clin Endocrinol Metab 2007;92:4514-21.

13. Williams KM. Update on bone health in pediatric chronic disease. Endocrinol Metab Clin North Am 2016;45:433-41.

14. Choudhary G, Gupta RK, Beniwal J. Bone mineral density in celiac disease. Indian J Pediatr 2016 Dec 27 [Epub]. https://doi.org/10.1007/s12098-016-2273-1.

15. Carnevale V, Romagnoli E, D'Erasmo E. Skeletal involvement in patients with diabetes mellitus. Diabetes Metab Res Rev 2004;20:196-204.

16. Galluzzi F, Stagi S, Salti R, Toni S, Piscitelli E, Simonini G, et al. Osteoprotegerin serum levels in children with type 1 diabetes: a potential modulating role in bone status. Eur J Endocrinol 2005;153:879-85.

17. Khan TS, Fraser LA. Type 1 diabetes and osteoporosis: from molecular pathways to bone phenotype. J Osteoporos 2015;2015:174186.

18. Wasserman H, O'Donnell JM, Gordon CM. Use of dual energy X-ray absorptiometry in pediatric patients. Bone 2016 Dec 15 [Epub]. https://doi.org/10.1016/ j.bone.2016.12.008.

19. Zemel BS, Kalkwarf HJ, Gilsanz V, Lappe JM, Oberfield $\mathrm{S}$, Shepherd JA, et al. Revised reference curves for bone mineral content and areal bone mineral density according to age and sex for black and non-black children: results of the bone mineral density in childhood study. J Clin Endocrinol Metab 2011;96:3160-9.

20. Crabtree NJ, Arabi A, Bachrach LK, Fewtrell M, El-Hajj Fuleihan G, Kecskemethy HH, et al. Dual-energy X-ray absorptiometry interpretation and reporting in children and adolescents: the revised 2013 ISCD Pediatric Official Positions. J Clin Densitom 2014;17:225-42.

21. Zemel BS. Quantitative computed tomography and computed tomography in children. Curr Osteoporos Rep 2011;9:284-90.

22. Wang KC, Wang KC, Amirabadi A, Cheung E, Uleryk E, Moineddin R, et al. Evidence-based outcomes on diagnostic accuracy of quantitative ultrasound for assessment of pediatric osteoporosis - a systematic review. Pediatr Radiol 2014;44:1573-87.

23. Weeks BK, Hirsch R, Nogueira RC, Beck BR. Is calcaneal broadband ultrasound attenuation a valid index of dualenergy $\mathrm{x}$-ray absorptiometry-derived bone mass in children? Bone Joint Res 2016;5:538-43.

24. Cimaz R, Stagi S. Managing pediatric osteoporosis. Int J Clin Rheumatol 2013;8:509-12

25. Zhang C, Liu Z, Klein GL. Overview of pediatric bone problems and related osteoporosis. J Musculoskelet Neuronal Interact 2012;12:174-82.

26. Andrews EB, Gilsenan A, Midkiff K, Harris D. Challenges in studying very rare cancer outcomes and infrequent exposures: example of teriparatide and osteosarcoma. Ann Epidemiol 2016;26:751-3. 